\title{
Estimation of image scale variations in monocular visual odometry systems
}

\begin{abstract}
Reliable estimation of the vehicle position is the main prerequisite in all autonomous mobile robotic applications. Image-scale uncertainty in correlation-based monocular visual odometry systems negatively affects the accuracy of the vehicle motion estimation. This paper presents the development of a new technique and algorithm to estimate image-scale variations due to camera height fluctuations when the vehicle is driven on uneven terrains or when the height of vehicle from ground changes as a result of changes in load or number of passengers in the vehicle. This technique depends on marking the image frames by two red laser points, as independent reference points, which have a certain distance between them. The image-scale variations can be estimated by monitoring the variations in the distance between these two reference points. The proposed technique eliminates the need for camera recalibration and the use of sensors to measure the variations of camera height from ground, such as laser range finders and acceleration sensors. The developed system uses a single downward-facing monocular camera supported by a lighting module and installed underneath the test vehicle to avoid the negative effect of directional sunlight and shadows, which can disturb the correlation. Indoor and outdoor experiments have proven the efficiency of the suggested technique in resolving image-scale uncertainty and ensuring an image-scale-invariant correlation-based matching, with only less than 5\% additional computational time.
\end{abstract}

Keyword: Image-scale uncertainty; Monocular visual odometry; Downward-facing camera; Image rescaling; Low-textured environments; Motion estimation 